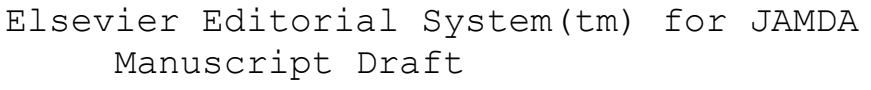

Manuscript Number: JAMDA-D-18-00172

Title: Consolidated Medication Review Algorithm to Improve Medications Use in Older Adults: Components, Scoring Scheme and Implementation

Article Type: Letter to the Editor

Keywords: Algorithm, Older Adults, Medication Review, Medications Use Corresponding Author: Dr. Shahzad Hasan Syed, PhD

Corresponding Author's Institution: International Medical University

First Author: Kaeshaelya Thiruchelvam, MSc

Order of Authors: Kaeshaelya Thiruchelvam, MSc; Pei Se Wong, PhD; Therese Kairuz, PhD; Zaheer Ud-Din Babar, PhD; Shahzad Hasan Syed, PhD 
Consolidated Medication Review Algorithm to Improve Medications Use in Older Adults: Components, Scoring Scheme and Implementation

\section{List of Authors}

Kaeshaelya Thiruchelvam

International Medical University, Malaysia

Email: kaeshaelya@imu.edu.my

Dr Pei Se Wong

International Medical University, Malaysia

Email: peise_wong@imu.edu.my

Associate Professor Therese Kairuz

University of Newcastle, Australia

Email: therese.kairuz@newcastle.edu.au

Professor Zaheer Ud-Din Babar

University of Huddersfield, UK

Email: z.babar@hud.ac.uk

Dr Syed Shahzad Hasan

Corresponding author

University of Huddersfield

Queensgate, HD1 4BX, Huddersfield, UK

Email: s.hasan@hud.ac.uk

Contact No: +447568497278 


\section{Consolidated Medication Review Algorithm to Improve Medications Use in Older Adults: Components, Scoring Scheme and Implementation}

\section{To the Editor:}

Medication review algorithms and minimisation frameworks are known to aid reviewers in identifying and discontinuing potentially inappropriate medications, which may otherwise lead older population to harmful events. ${ }^{1}$ There have been many algorithms and minimisation frameworks that have been developed to aid to evaluate medication reviews, with each one offering benefits in various settings. In 2007, Pollock et al. proposed an eight-step approach to improve prescribing by family physicians. ${ }^{2}$ Scott et al. (2012) further developed the concept proposed by Pollock et al. (2007) to a ten-step framework that aimed to improve medicines use and to reduce the number of inappropriate medications prescribed to older people. ${ }^{3}$ Poudel et al. (2015) further condensed the ten-step framework to a four-step algorithm that guides clinicians in reviewing the medications, but it is targeted at frail older patients living in aged care facilities. ${ }^{1}$ Many other independent models and algorithms have also been designed. ${ }^{4-6} \mathrm{~A}$ commonality that exists among all medication review algorithms and minimisation frameworks is that all of them lack scoring scheme to quantify medication review process. In this context, a new consolidated medication review algorithm is being developed.

\section{Consolidated Medication Review Algorithm}

Our algorithm is different from the previous algorithms in two ways, first it incorporates the potentially inappropriate medication (PIM) component, second it includes a scoring scheme which correspond to the components in the algorithm (Figure 1). The scores derived from the algorithm will allow for an effective periodical review to observe improvements in medicine use.

\section{Components of an algorithm}

The components in our version of medication review algorithm are: ascertain and validate indications of the medications (consult prescriber and credible resources like Micromedex®); identify therapeutic duplications (review complete list of current medications); ascertain the effectiveness of the medications (cross-referenced via objective measurements as per patient records, or obtained via clarification with the respective prescriber); perform a two-stage screening of the medications; 4a. Identify potentially inappropriate medications (consider Tables 2 and 3 of 2015 Beers Criteria, ${ }^{7}$ and/ or START/STOPP criteria $^{8}$ ); 4 b. Identify medications to be 
used with caution (consider Table 4 of 2015 Beers Criteria $^{7}$ ), appropriateness of the dosing regimen (consider Table 5 of 2015 Beers Criteria, ${ }^{7}$ or credible resources, e.g. Micromedex ${ }^{\circledR}$, and consult prescriber), contraindications (consider resources e.g. Micromedex®), incidence of adverse drug events (consider Table 6 on drug interactions from 2015 Beers Criteria; ${ }^{7}$ ADRs consider Naranjo adverse drug reaction probability scale ${ }^{9}$ ), consider continuing, discontinuing or substituting medications (consult prescriber). If the medicine is deemed ineffective for the patient, then the medication is warranted for discontinuation, in which the withdrawal regimen (consider Table developed by Poudel et al. ${ }^{1}$ ) or non-pharmacological interventions should be considered.

\section{Scoring scheme}

The scores are generated in a similar manner like Medication Appropriateness Index (MAI). ${ }^{10}$ According to scoring scheme, appropriate items are scored 0 and inappropriate responses are scored 1. The weighting scheme combines all the criteria, and they are as follows: 3 for indication, effectiveness, use of PIM and drug-disease interactions; 2 for dosage, directions and drug-drug interactions; and 1 for duplication, duration and expense. A higher single score means increased medication inappropriateness (possible score range of 0-21). A medication is deemed inappropriate overall and should be discontinued if the medication scored a rating of 3 or above. The ADR component was excluded from the scoring scheme, mainly due to uncertainty of causality. It may not be feasible to determine the cause of an ADR instantaneously and would require further clarification.

\section{Implementation}

We implemented this algorithm in a 6-month prospective study among 202 residents aged 65 years and above across 17 aged care facilities in Malaysia (Ethics approval was obtained Project IDIA422, Research Code, XX University, Kuala Lumpur, Malaysia 349/2016). The proportions of older adults exposed to inappropriate medications detected by our scoring scheme that were not detected by the MAI were $39.7 \%$ at baseline, $34.8 \%$ at 3 -months and $34.3 \%$ at 6 months. Conversely the proportion of older adults exposed to inappropriate medications detected by the MAI that were not detected by our scoring scheme were almost none or close to minimal $(0.0 \%$ at baseline and at 6 -months, and $3.5 \%$ at 3 -months). It is also noteworthy that the incorporation of the PIMs is crucial in a medication review algorithm for the older people as 
there are many benefits when PIMs are avoided in this population. The medication review should be objective in nature, also a review with stepwise approach would allow easy implementation.

\section{References}

1. Poudel A, Ballokova A, Hubbard RE, Gray LC, Mitchell CA, Nissen LM, et al. Algorithm of medication review in frail older people: Focus on minimizing the use of high-risk medications. Geriatr Gerontol Int 2015 Sep 3; 16(9): 1002-13.

2. Pollock M, Bazaldua OV, Dobbie AE. Appropriate prescribing of medications: an eightstep approach. Am Fam Physician 2007 Jan 15; 75(2): 231-6.

3. Scott IA, Gray LC, Martin JH, Mitchell CA. Minimizing inappropriate medications in older populations: a 10-step conceptual framework. Am J Med 2012 Jun; 125(6): 529_ 37.e4.

4. Holmes HM, Hayley DC, Alexander GC, Sachs GA. Reconsidering medication appropriateness for patients late in life. Arch Intern Med 2006 Mar 27; 166(6): 605-9,

5. Jetha S. Polypharmacy, the Elderly, and Deprescribing. Consult Pharm J Am Soc Consult Pharm 2015 Sep; 30(9): 527-32.

6. Hilmer SN, Gnjidic D, Le Couteur DG. Thinking through the medication list appropriate prescribing and deprescribing in robust and frail older patients. Aust Fam Physician 2012 Dec; 41(12): 924-8.

7. American Geriatrics Society 2015 Beers Criteria Update Expert Panel. American Geriatrics Society 2015 Updated Beers Criteria for Potentially Inappropriate Medication Use in Older Adults. J Am Geriatr Soc 2015 Nov; 63(11): 2227-46.

8. O’Mahony D, Gallagher P, Ryan C, Byrne S, Hamilton H, Barry P, et al. STOPP \& START criteria: A new approach to detecting potentially inappropriate prescribing in old age. Eur Geriatr Med 2010 Feb; 1(1): 45-51.

9. Naranjo CA, Busto U, Sellers EM, Sandor P, Ruiz I, Roberts EA, et al. A method for estimating the probability of adverse drug reactions. Clin Pharmacol Ther 1981 Aug; 30(2): 239-45.

10. Hanlon JT, Schmader KE, Samsa GP, Weinberger M, Uttech KM, Lewis IK, et al. A method for assessing drug therapy appropriateness. J Clin Epidemiol 1992 Oct; 45(10): $1045-51$. 


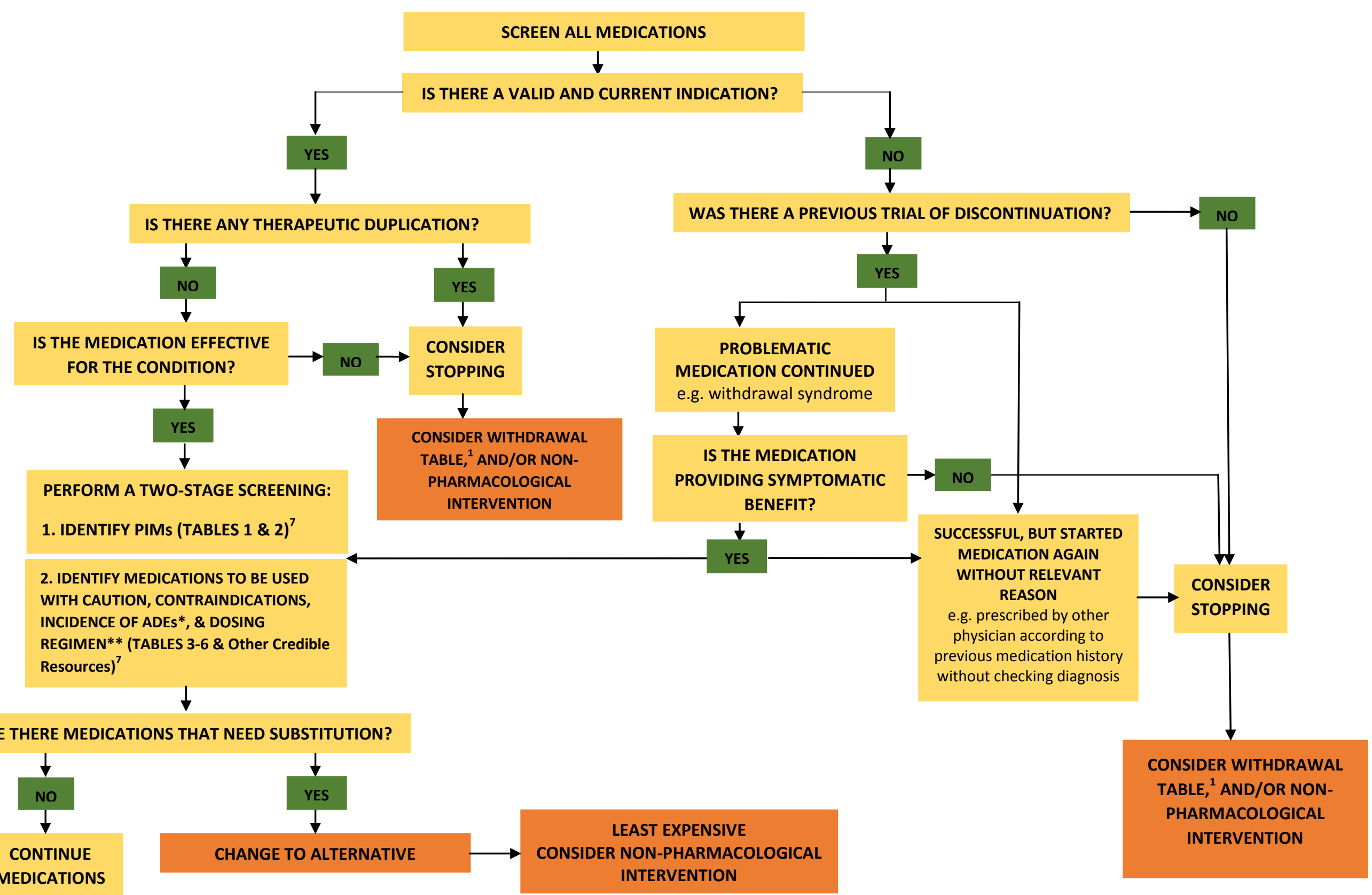

*ADE COMPRISES DRUG INTERACTIONS \& ADVERSE DRUG REACTIONS 